\title{
Physical interaction and assembly of Bacillus subtilis spore coat proteins CotE and CotZ studied by atomic force microscopy
}

Huiqing Liu ${ }^{\text {a,b,1 }}$, Haiyan Qiao ${ }^{\text {c,1 }}$, Daniela Krajcikova ${ }^{\text {d,1 }}$, Zhe Zhang ${ }^{\text {a }}$,

Hongda Wang ${ }^{\text {a }}$, Imrich Barak ${ }^{\text {d }}$, Jilin Tang ${ }^{\mathrm{a}, *}$ 

The spore
multilayered
and essen
of the co
challenge.
proteins a
based sin
interaction
CotZ. The
between

\section{Abstract}

The spore of Bacillus subtilis, a dormant type of cell, is surrounded by a complex multilayered protein structure known as the coat. It is composed of over 70 proteins and essential for the spore to withstand extreme environmental conditions and allow germination under favorable conditions. However, understanding how the properties of the coat arise from the interactions among all these proteins is an important challenge. Moreover, many specific protein-protein interactions among the coat proteins are crucial for coat assembly. In this study, atomic force microscopy (AFM) based single molecule force spectroscopy (SMFS) was applied to investigate the interaction as a dynamic process between two morphogenetic coat proteins, CotE and CotZ. The unbinding force and kinetic parameters characterizing the interaction

\section{Keywords}

Atomic force microscopy, single molecule force spectroscopy, protein-protein interaction, self-assembly, $\operatorname{CotE}, \operatorname{Cot} Z$ 


\section{Introduction}

The formation of Bacillus subtilis spores, a highly dormant cell type, is triggered by condition of nutrient limitation. Spores are able to withstand extremes of temperature, radiation, chemical assault and even the vacuum of outer space (Driks, 1999; Nicholson et al., 2000). The capability of the spores to withstand harsh environments is in large part due to the spore coat, a thick proteinaceous shell. The coat comprises no less than 70 individual proteins, organized into four main structural layers: a diffuse undercoat, a laminated lightly staining inner layer, a thick, highly electron dense outer layer and an outermost surface part of the coat called a crust (McKenney et al., 2013).

The assembly of the coat layers is controlled by a subset of coat proteins, known as the morphogenetic proteins (Henriques and Moran, 2007), including SpoIVA, SpoVID, SafA, CotE, and CotZ. They have no impact on coat protein gene expression but act post-translationally to direct the assembly of the various coat components around the forming spore (Kim et al., 2006). SpoIVA which assembles in the coat basement layer with the assistance of SpoVM, a small amphipathic $\alpha$-helical peptide, plays a key role in localization of other coat protein components on the spore surface (Ramamurthi et al., 2006). SpoVID along with SpoVM are necessary for the spore encasement during which the spore becomes fully surrounded by coat proteins (de Francesco et al., 2012; Wang et al., 2009). SafA is the major inner coat morphogenetic protein and contributes to patterning of the coat structure (McKenney et al., 2010; Ozin et al., 2000; Ozin et al., 2001). The morphogenetic coat protein 
CotE is localizing at the junction of the inner and outer coat layers, forming a ring around the forespore (Driks et al., 1994; Little and Driks, 2001). It regulates the assembly of a large set of coat proteins, most of the outer coat proteins, such as CotZ, $\operatorname{Cot} \mathrm{A}, \operatorname{Cot} \mathrm{B}, \operatorname{Cot} \mathrm{C}, \operatorname{CotH}, \operatorname{Cot} M, \operatorname{Cot} U$ and $\operatorname{Cot} \mathrm{G}$ (Donovan et al., 1987; Zheng et al., 1988). A series of mutagenesis studies indicated that CotE has a modular structure with C-terminal region that directs various coat proteins assembly (Bauer et al., 1999). CotZ is acting as a morphogenetic protein and was identified to be the component of the outermost layer of the coat named the crust (Imamura et al., 2011; McKenney et al., 2010).

Coat assembly in B. subtilis is serving as an excellent tractable model for investigation of the self-assembly process of biological structures that has been studied for decades (Henriques and Moran, 2000; Piggot and Coote, 1976; Pogliano et al., 1995). These studies clarified the key role of morphogenetic proteins in formation of the coat structure. In addition, it was suggested that specific protein-protein interactions determine the manner how structural components of the coat are deposited within the coat (Costa et al., 2006; Muellerova et al., 2009). However, the interactions among the over 70 proteins making up the coat remain largely obscure.

Over the past decades genetic and biochemical techniques has been employed for identification of non-covalent interactions among spore-coat proteins (Isticato et al., 2010; Krajcikova et al., 2009; Muellerova et al., 2009; Zhang et al., 1993; Zilhao et al., 2004). Although combining of these two basic approaches allows revealing a number of protein-protein interactions, the sensitivity and specificity of classical methods are 
relatively low (Krajcikova et al., 2009). Recently atomic force microscopy (AFM) has become an extremely versatile tool to investigate morphological and mechanical properties of biological materials at the nanoscale in their near-native environment (Fotiadis et al., 2002; Hinterdorfer and Dufrene, 2006; Plomp et al., 2002; Shao et al., 1996). Furthermore, AFM based single molecule force spectroscopy (SMFS) allows the detection of unbinding forces and the dynamic processes of biological pairs at the single-molecule level (Cecchet et al., 2008; Chtcheglova et al., 2010; Dufrene and Hinterdorfer, 2008; Tang et al., 2009; Wollschlaeger et al., 2009; Zhu et al., 2010), owing to its capability of piconewton force sensitivity and nanometer positional accuracy. Therefore, the examination of the interactions among spore coat proteins by single molecule force spectroscopy is one of the most powerful approaches for visualizing the interactions (Liu et al., 2015; Qiao et al., 2012; Qiao et al., 2013).

Previous work revealed that CotE could interact with most or all the outer coat components (Kim et al., 2006). In addition, pull-down assay indicated that a low-affinity interaction between CotE and CotZ exists (Krajcikova et al., 2009). Another indication of their interaction was acquired from a genetic approach (Kim et al., 2006; McKenney and Eichenberger, 2012; McKenney et al., 2010; Plomp et al., 2014). In this study, we investigate $\operatorname{Cot} E$ and $\operatorname{Cot} Z$ interactions and self-assembly characteristics of these proteins. The unbinding forces of the $\operatorname{Cot} E-\operatorname{Cot} Z$ complex, which characterize the interaction strength between two proteins, were investigated by SMFS. Additionally, by measuring the unbinding force of the complex at different values of loading rate the binding kinetics was also studied. We further applied AFM 
imaging to characterize the assembly behaviors of CotE and CotZ, individually or in combination at solid-liquid interfaces. These studies could be extended to other spore coat proteins, which can provide insight into the complex network of interactions and assembly of the spore coat.

\section{Experimental section}

\subsection{Materials}

Silicon wafers were purchased from Institute of Microelectronics, Peking University. 3-aminopropyltriethoxysilane (APTES), triethylamine and ethanolamine were obtained from Sigma-Aldrich. $\mathrm{NaCNBH}_{3}$ was obtained from J\&K Scientific Ltd. NHS-PEG 18 -aldehyde was obtained from Prof. Hermann J. Gruber (Johannes Kepler University, Austria). Other reagents used in all experiments were of analytical grade. Milli-Q-purified water (18.2 M $\Omega$ ) was used for all solution preparations.

\subsection{Protein expression and purification}

Escherichia coli BL21(DE3) strain harboring the plasmids pEtcotE or pETDuetcotZ were used for protein production. Bacteria were grown at $37{ }^{\circ} \mathrm{C}$ and protein expression was induced by $1 \mathrm{mM}$ IPTG. His-tagged recombinant proteins were isolated and purified exactly as described previously (Krajcikova et al., 2009) using metal affinity chromatography on $\mathrm{Ni}^{2+}$ column in Tris buffer containing $8 \mathrm{M}$ urea.

\subsection{The dialysis procedure for $\operatorname{Cot} E$ and $\operatorname{Cot} Z$}

The purified proteins (CotE and CotZ) at a concentration of $100 \mu \mathrm{g} / \mathrm{mL}$ were dialyzed against 3 litres of water for 3 hours at room temperature, during which the 
water was changed every hour. After dialysis, the proteins were centrifuged for 7 minutes at $4{ }^{\circ} \mathrm{C}$ at $14000 \mathrm{rpm}$ to remove protein aggregates and unsolved particles. Finally, the proteins were diluted to different concentration with phosphate-buffered saline (PBS) buffer $\left(1.8 \mathrm{mM} \mathrm{KH}{ }_{2} \mathrm{PO}_{4}, 8.1 \mathrm{mM} \mathrm{Na} 2 \mathrm{HPO}_{4}, 2.7 \mathrm{mM} \mathrm{KCl}, 140 \mathrm{mM}\right.$ $\mathrm{NaCl}, \mathrm{pH} 7.5)$.

\subsection{Silicon substrates and AFM tips preparation}

The silicon wafers were cut into $0.7 \mathrm{~cm} \times 0.7 \mathrm{~cm}$ pieces. Prior to functionalization, the silicon pieces were cleaned with a freshly prepared piranha solution $\left(98 \% \mathrm{H}_{2} \mathrm{SO}_{4}: 30 \% \mathrm{H}_{2} \mathrm{O}_{2}=7: 3\right.$, v/v, caution: piranha solution is extremely explosive and must be treated with great care), followed by extensive rinsing with large amounts of water. Then, the silicon pieces were ultrasonically cleaned with water (3x), ethanol (3x), and dried under a nitrogen flow.

For SMFS, the general process for functionalization of the silicon pieces with CotE was similar to a previously reported procedure (Zhang et al., 2014). In brief, the cleaned silicon pieces were moved into an argon-gas-filled desiccator and incubated with $50 \mu \mathrm{L}$ 3-aminopropyltriethoxysilane (APTES) and $15 \mu \mathrm{L}$ triethylamine for 2 hours using a vapor-phase deposition method (Ebner et al., 2007). This step was referred to as silylation, which generated the required amino group density on the surface of the silicon pieces. Then, attachment of a flexible heterobifunctional polyethylene glycol (PEG) linker, NHS-PEG 18 -aldehyde, to the amino-functionalized silicon pieces via NHS reactive end was carried out by incubating the silicon pieces with PEG linker (2 mg/mL in chloroform containing $0.5 \%$ (v/v) triethylamine) for 2 
hours at room temperature. Following the reaction, the silicon pieces were cleaned thoroughly in chloroform to remove non-reacted PEG linkers. Subsequently, CotE was attached to the aldehyde end of the PEG linker via its lysine residues by mixing the activated silicon pieces with $100 \mu \mathrm{g} / \mathrm{mL}$ CotE protein solution containing freshly prepared $20 \mathrm{mM} \mathrm{NaCNBH}$ for 1 hour at room temperature (Zhu et al., 2010). Finally, the unoccupied aldehyde groups on the silicon substrate were blocked by the addition of ethanolamine (final concentration of $25 \mathrm{mM}, \mathrm{pH} 9.6$, preadjusted with $\mathrm{NaOH}$ ) for 30 minutes. After the reaction, the prepared silicon substrates were rinsed with PBS buffer and stored in the buffer at $4{ }^{\circ} \mathrm{C}$ until use.

Silicon nitride AFM tips (MSCT, Veeco, USA) were used for force spectroscopy studies. Following the same steps as above, AFM tips were functionalized with CotZ. However, AFM tips for control experiments were modified only with a PEG linker whose terminal aldehyde group had been inactivated by incubating with $25 \mathrm{mM}$ ethanolamine for 30 minutes.

\subsection{AFM measurements}

The AFM samples were prepared by applying $50 \mu \mathrm{L}$ of proteins with various concentrations onto the cleaned silicon substrates following the incubation for 5 hours at room temperature and then stored overnight at $4{ }^{\circ} \mathrm{C}$. AFM imaging was carried out on NanoScope Multimode 8 (Digital Instruments, Veeco, USA) in PBS buffer at room temperature. The images were captured in Tapping Mode, using commercial $\mathrm{Si}_{3} \mathrm{~N}_{4}$ tips (DNP-S10, $0.06 \mathrm{~N} / \mathrm{m}$, Veeco, USA). The scan rate for imaging was set at 1-2 lines/s in the $512 \times 512$ pixel format. The acquired images were analyzed using 
NanoScope Analysis (Veeco, USA). A PicoSPM 5500 AFM (Agilent Technologies, Andover, USA) was used to perform all SMFS experiments in PBS buffer at room temperature. Force measurements were carried out with CotE-functionalized silicon substrates and CotZ-modified tips. More than 2000 force-distance curves were obtained at randomly chosen five to eight locations on silicon surface. The experiment was repeated three times with different CotZ-modified AFM tips. Data analysis of the selected thousands of curves was performed with Matlab Version 7 (MathWorks, Natick, MA) as previously described (Baumgartner et al., 2000b). Empirical probability density functions (pdf) calculated as described earlier was applied to present the force distributions of the unbinding events (Baumgartner et al., 2000a; Riener et al., 2003). The spring constant of every cantilever was calibrated after a given experiment by the thermal-noise mode in air (Butt and Jaschke, 1995; Hutter and Bechhoefer, 1993). The loading rate $r$, was determined by using the equation $r=$ $v k_{\text {eff, }}$ with $v$ being the cantilever retraction rate and $k_{\text {eff }}$ being the effective spring constant (De Paris et al., 2000).

\section{Results and discussion}

\subsection{Single molecule force spectroscopy study of the CotE-CotZ interaction}

AFM based SMFS was used to investigate the interaction between CotE and CotZ at the single-molecule level. To perform this experiment, CotZ was covalently coupled to the amino-functionalized AFM tip and CotE was immobilized onto the amino-modified silicon substrate through a flexible heterobifunctional PEG linker (Fig. 1a). Attributed to the nonlinear stretching elasticity of the PEG linkers, it is easy 
to discriminate between specific and nonspecific unbinding events in the force-distance cycles (Hinterdorfer and Dufrene, 2006). The force-distance curves were obtained by approaching and subsequently retracing the AFM tip with CotZ from the silicon substrate, on which CotE was immobilized. A typical force-distance curve with a single molecular recognition event between $\operatorname{Cot} E$ and $\operatorname{Cot} Z$ is depicted in Fig. 1b. The peak in the retracting curve, which emerged about $13 \mathrm{~nm}$ away from the separation of the tip and substrate, represents the specific unbinding event between CotE and CotZ. The characteristic nonlinear force signal in the retracting curve originates from the stretching elasticity of the PEG linker (Wildling et al., 2012). In order to confirm that CotE could specifically recognize CotZ, a control experiment was carried out by using an AFM tip functionalized only with the PEG linker. As shown in Fig. 1b, inset, the specific recognition event disappeared in the retracting curve. The probability density function (pdf) of the unbinding forces obtained from about 2000 force-distance curves shows the distribution of unbinding forces between CotE and $\operatorname{CotZ}$ (Fig. 1c). The maximum of the distribution was $40.9 \pm 0.1 \mathrm{pN}$ at the loading rate of $1.12 \times 10^{3} \mathrm{pN} / \mathrm{s}$, reflecting the most probable unbinding force $\left(f_{\mathrm{u}}\right)$ of CotE-CotZ complex. The binding probability (probability of detecting specific recognition events in all force-distance cycles) was $13.6 \%$. However, in the control experiment, the binding probability remarkably decreased to $3.4 \%$. These data clearly prove that CotE immobilized on the silicon substrates could be specifically detected at the single-molecule level by $\operatorname{CotZ}$ attached to AFM tips and that this interaction is specific. 
The unbinding forces of the receptor-ligand complex rely not only on the interaction of molecules but also on the loading rate of the AFM tip (Merkel et al., 1999). According to the Bell-Evans model (Bell, 1978) there is a linear relationship between the individual unbinding force $f_{\mathrm{u}}$ and the logarithm of the loading rate $r$ following Equation (1):

$$
f_{\mathrm{u}}=\left(\frac{k_{\mathrm{B}} T}{x_{\beta}}\right) \ln \left(\frac{r x_{\beta}}{k_{\mathrm{B}} T k_{\mathrm{off}}}\right)
$$

where $x_{\beta}$ is the difference of the energy barrier to the minimum energy along the separation path, $k_{\text {off }}$ is the dissociation rate constant at zero force, $k_{\mathrm{B}}$ is the Boltzmann constant, $T$ is the thermodynamic temperature.

The dynamic force measurement of the $\operatorname{CotE}-\operatorname{Cot} Z$ complex was carried out at different loading rates from $3.5 \times 10^{2}$ to $4.9 \times 10^{3} \mathrm{pN} / \mathrm{s}$. As illustrated in Fig. 2, the unbinding forces of the CotE-CotZ complex varied in the range of $38-102 \mathrm{pN}$ and increased linearly with increasing logarithm of the loading rate. Based on the slope and the intercept of the fitting curves (Fig. 2), the separation of energy barrier from the dissociated state to bound state of the CotE-CotZ complex $x_{\beta}$ was $0.836 \pm 0.006 \AA$, the kinetic off-rate constant $k_{\text {off }}$ and bond-survival time $\tau\left(=1 / k_{\text {off }}\right)$ were $0.122 \pm 0.001$ $\mathrm{s}^{-1}$ and $8.19 \pm 0.05 \mathrm{~s}$, respectively. The interaction forces between these proteins and kinetic rate constants obtained from SMFS characterize the interaction strength of protein pairs. Based on the comparisons of the interaction forces and the dissociation rates, the stability of the complexes could be deduced. It was found that at the same loading rate, dissociation of CotZ from CotE requires higher unbinding forces than 
CotY from CotE, which reveals that the $\operatorname{Cot} E-\operatorname{CotZ}$ interaction is stronger than CotE-CotY. Previous studies showed that the interactions of $\operatorname{CotE}$ with $\operatorname{Cot} Y$ could be regarded as the strong one (Liu et al., 2016). Thus, it can be concluded that CotE displays a high binding affinity to CotZ to form a stable complex. Moreover, the CotE-CotZ complex has similar dissociation rate and lifetime to CotE-Cot $\mathrm{Y}$ complex, indicating that the stability of CotE-CotZ complex is high. All these results indicate that there is a strong affinity between CotE and CotZ. These AFM measurements also reflect that the localization of $\operatorname{CotZ}$ on the spore surface is directly controlled by $\operatorname{CotE}$ (Kim et al., 2006).

\subsection{Assembly of CotE and CotZ on silicon substrate in vitro}

The study of CotE and CotZ assembly behavior in vitro might help understand a small fraction of complex biological process of formation highly organized spore coat. To obtain some basic information about their assembly, we first performed AFM to characterize the morphology of CotE and CotZ individually assembling on silicon substrate. The representative AFM topographic images at different scan sizes of CotE and CotZ are presented in Fig. 3. It can be observed that CotE self-assembled into extended net-like structures (Fig. $3 \mathrm{a}_{1}$, white arrows). At higher magnification, the mesh sizes (Fig. 3a $\mathrm{a}_{2}$, white arrows) were measured in a range from 40 to $90 \mathrm{~nm}(n=$ 20). These results are in good agreement with electron micrographs of typical CotE aggregates, displaying extended net-like structures (Jiang et al., 2015). However, the average repeat spacing was about $15 \mathrm{~nm}$, although the mesh size was highly variable. When CotZ assembled on silicon substrate, ordered strip structures were visualized 
(Fig. $3 b_{1}$, black arrows). To observe more details, a portion of Fig. $3 b_{1}$ was magnified as shown in Fig. $3 b_{2}$. The strips we observed were arranged in parallel to each other and also made contacts with adjacent strips. The average width of parallel strips is $15-35 \mathrm{~nm}(n=20)$.

Since CotE, one of the most important morphogenetic proteins, could direct the assembly of most of the outer coat proteins, we then investigated the assembly behaviors of CotE in complex with $\operatorname{CotZ}$ at different CotE/CotZ ratios $(3: 1,2: 1,1: 2$ and 1:3). Fig. 4 shows typical AFM images of the complex of CotE with CotZ formed at different molar ratios, which were different from the morphologies when individual CotE and CotZ proteins were applied. We found out that there is extensive network of filaments in cases of $\operatorname{CotE} / \operatorname{Cot} Z$ ratio $3: 1$ and 2:1 (Fig. $4 a_{1}$ and $b_{1}$ ). The primary difference between these two images is the size of the mesh. The mesh sizes (white arrows) in Fig. $4 \mathrm{a}_{2}$ distribute in the range of $15-40 \mathrm{~nm}(n=30)$, which are bigger than that in Fig. $4 b_{2}$. However, when the CotE/CotZ ratio is decreased from $2: 1$ to $1: 2$, the complex assembled from initial mesh-like structure to parallel strips (black arrows) with an average width of 5-20 nm $(n=20)$ and some also stuck together (Fig. $4 \mathrm{c}_{1}$ and $\left.4 \mathrm{c}_{2}\right)$. The width of most strips became greater than $40 \pm 5 \mathrm{~nm}\left(\right.$ Fig. $4 \mathrm{~d}_{1}$ and $\left.4 \mathrm{~d}_{2}, n=15\right)$ when the ratio of $\operatorname{Cot} E / \operatorname{Cot} Z$ was kept at 1:3. The morphology investigation indicates that CotE-CotZ assembly is dependent on their molar ratios. That is, at high $\mathrm{CotE} / \mathrm{CotZ}$ ratio, the complex prefers to form extensive mesh-like structure, and a low ratio of CotE to CotZ facilitates the formation of parallel strips. 
The interaction forces and dynamic data of CotE-CotZ complex demonstrate that CotE has a strong affinity for $\operatorname{Cot} Z$ to form a stable complex. The changes in morphology of CotE-CotZ assembly may be attributed to the conformational changes of $\operatorname{CotE}$ and $\operatorname{CotZ}$ when they interact with each other. Importantly, it is tempting to hypothesize that the two-dimensional nets formed by CotE encapsulate the forespore and serve as a platform for the assembly of CotZ. Our study provides further evidence that CotE can directly interact with CotZ and this can contribute to the attachment of CotZ onto the spore surface allowing the crust firmly adhering to the spore. Therefore, this work has an important contribution to better understand the relationship between the assembly and interaction of the spore coat proteins.

\section{Conclusion}

The spore coat of $B$. subtilis represents a unique example of macromolecular assemblies. Like many other examples of morphogenesis in biology, the coat assembly is a dynamic and complex multistep process. The protein interactions between the coat proteins, especially morphogenetic proteins are pinpointing factor which determines the coat assembly process. Thus, the specific interaction between CotE and CotZ studied here refines our perception about the principles which govern the morphogenetic proteins to create a basic scaffold where other coat proteins are attached within the process of spore coat development. Using the AFM based SMFS the unbinding force and kinetic data characterizing the interaction of $\operatorname{Cot} E$ and $\operatorname{Cot} Z$ was obtained. We demonstrated that the morphogenetic protein CotE could interact directly and specifically with CotZ forming a stable complex. Moreover, AFM 
imaging was performed to study the assembly behaviors of $\operatorname{CotE}$ and $\operatorname{CotZ}$ at solid-liquid interfaces. Our results showed that at high CotE/CotZ ratio, the complex prefers to form extensive mesh-like structure, and a low ratio of $\operatorname{CotE}$ to $\operatorname{CotZ}$ facilitates the formation of parallel strips. Together with our previous results obtained using this method we gradually are getting the more accurate perception of the assembly and properties of the spore coat. This work clearly shows unique advantages of the SMFS technique for future characterization of other coat proteins.

\section{Acknowledgements}

This work was supported by the National Science Foundation of China (No. 21275140, 21405152), the Science and Technology Development Plan of Jilin Province (20130101126JC), VEGA Grant 2/0131/14 from the Slovak Academy of Sciences to D.K. and a grant from the Slovak Research and Development Agency (APVV-14-0181) to I.B. We gratefully thank Dr. Christian Rankl for the help in analyzing the data. 


\section{References}

Bauer, T., Little, S., Stover, A.G., Driks, A., 1999. Functional regions of the Bacillus subtilis spore coat morphogenetic protein CotE. J. Bacteriol. 181, 7043-7051.

Baumgartner, W., Hinterdorfer, P., Schindler, H., 2000a. Data analysis of interaction forces measured with the atomic force microscope. Ultramicroscopy 82, 85-95.

Baumgartner, W., Hinterdorfer, P., Ness, W., Raab, A., Vestweber, D., Schindler, H., Drenckhahn, D., 2000b. Cadherin interaction probed by atomic force microscopy. Proc. Natl. Acad. Sci. U. S. A. 97, 4005-4010.

Bell, G.I., 1978. Models for specific adhesion of cells to cells. Science 200, 618-627.

Butt, H.J., Jaschke, M., 1995. Calculation of thermal noise in atomic-force microscopy. Nanotechnology 6, 1-7.

Cecchet, F., Lussis, P., Jerome, C., Gabriel, S., Silva-Goncalves, E., Jerome, R., Duwez, A.-S., 2008. A generic chemical platform for molecular recognition and stimuli-responsive probes based on scanning probe microscopy. Small 4, $1101-1104$.

Chtcheglova, L.A., Wildling, L., Waschke, J., Drenckhahn, D., Hinterdorfer, P., 2010. AFM functional imaging on vascular endothelial cells. J. Mol. Recognit. 23, 589-596.

Costa, T., Isidro, A.L., Moran, C.P., Jr., Henriques, A.O., 2006. Interaction between coat morphogenetic proteins SafA and SpoVID. J. Bacteriol. 188, 7731-7741.

de Francesco, M., Jacobs, J.Z., Nunes, F., Serrano, M., McKenney, P.T., Chua, M.-H., Henriques, A.O., Eichenberger, P., 2012. Physical interaction between coat 
morphogenetic proteins SpoVID and CotE is necessary for spore encasement in Bacillus subtilis. J. Bacteriol. 194, 4941-4950.

De Paris, R., Strunz, T., Oroszlan, K., Güntherodt, H.-J., Hegner, M., 2000. Force spectroscopy and dynamics of the biotin-avidin bond studied by scanning force microscopy. Single Mol. 1, 285-290.

Donovan, W., Zheng, L., Sandman, K., Losick, R., 1987. Gene encoding spore coat polypeptides from Bacillus subtilis. J. Mol. Biol. 196, 1-10.

Driks, A., 1999. Bacillus subtilis spore coat. Microbiol. Mol. Biol. Rev. 63, 1-20.

Driks, A., Roels, S., Beall, B., Moran, C.P., Losick, R., 1994. Subcellular-localization of proteins involved in the assembly of the spore coat of Bacillus subtilis. Genes Dev. 8, 234-244.

Dufrene, Y.F., Hinterdorfer, P., 2008. Recent progress in AFM molecular recognition studies. Pflug. Arch. Eur. J. Phy. 456, 237-245.

Ebner, A., Wildling, L., Kamruzzahan, A.S.M., Rankl, C., Wruss, J., Hahn, C.D., Hoelzl, M., Zhu, R., Kienberger, F., Blaas, D., Hinterdorfer, P., Gruber, H.J., 2007. A new, simple method for linking of antibodies to atomic force microscopy tips. Bioconjugate Chem. 18, 1176-1184.

Fotiadis, D., Scheuring, S., Muller, S.A., Engel, A., Muller, D.J., 2002. Imaging and manipulation of biological structures with the AFM. Micron 33, 385-397.

Henriques, A.O., Moran, C.P., 2000. Structure and assembly of the bacterial endospore coat. Methods 20, 95-110.

Henriques, A.O., Moran, C.P., Jr., 2007. Structure, assembly, and function of the spore 
surface layers. Annu. Rev. Microbiol. 61, 555-588.

Hinterdorfer, P., Dufrene, Y.F., 2006. Detection and localization of single molecular recognition events using atomic force microscopy. Nat. Methods 3, 347-355.

Hutter, J.L., Bechhoefer, J., 1993. Calibration of atomic-force microscope tips. Rev. Sci. Instrum. 64, 1868-1873.

Imamura, D., Kuwana, R., Takamatsu, H., Watabe, K., 2011. Proteins involved in formation of the outermost layer of Bacillus subtilis spores. J. Bacteriol. 193, 4075-4080.

Isticato, R., Pelosi, A., De Felice, M., Ricca, E., 2010. CotE binds to CotC and CotU and mediates their interaction during spore coat formation in Bacillus subtilis. J. Bacteriol. 192, 949-954.

Jiang, S., Wan, Q., Krajcikova, D., Tang, J., Tzokov, S.B., Barak, I., Bullough, P.A., 2015. Diverse supramolecular structures formed by self-assembling proteins of the Bacillus subtilis spore coat. Mol. Microbiol. 97, 347-359.

Kim, H., Hahn, M., Grabowski, P., McPherson, D.C., Otte, M.M., Wang, R., Ferguson, C.C., Eichenberger, P., Driks, A., 2006. The Bacillus subtilis spore coat protein interaction network. Mol. Microbiol. 59, 487-502.

Krajcikova, D., Lukacova, M., Mullerova, D., Cutting, S.M., Barak, I., 2009. Searching for protein-protein interactions within the Bacillus subtilis spore coat. J. Bacteriol. 191, 3212-3219.

Little, S., Driks, A., 2001. Functional analysis of the morphogenetic spore coat Bacillus subtilis protein CotE. Mol. Microbiol. 42, 1107-1120. 
Liu, H., Krajcikova, D., Zhang, Z., Wang, H., Barak, I., Tang, J., 2015. Investigating interactions of the Bacillus subtilis spore coat proteins CotY and CotZ using single molecule force spectroscopy. J. Struct. Biol. 192, 14-20.

Liu, H., Krajcikova, D., Wang, N., Zhang, Z., Wang, H., Barak, I., Tang, J., 2016. Forces and kinetics of the Bacillus subtilis spore coat proteins $\operatorname{Cot} Y$ and $\operatorname{CotX}$ binding to CotE inspected by single molecule force spectroscopy. J. Phys. Chem. B 120, 1041-1047.

McKenney, P.T., Eichenberger, P., 2012. Dynamics of spore coat morphogenesis in Bacillus subtilis. Mol. Microbiol. 83, 245-260.

McKenney, P.T., Driks, A., Eichenberger, P., 2013. The Bacillus subtilis endospore: assembly and functions of the multilayered coat. Nat. Rev. Microbiol. 11, 33-44.

McKenney, P.T., Driks, A., Eskandarian, H.A., Grabowski, P., Guberman, J., Wang, K.H., Gitai, Z., Eichenberger, P., 2010. A distance-weighted interaction map reveals a previously uncharacterized layer of the Bacillus subtilis spore coat. Curr. Biol. 20, 934-938.

Merkel, R., Nassoy, P., Leung, A., Ritchie, K., Evans, E., 1999. Energy landscapes of receptor-ligand bonds explored with dynamic force spectroscopy. Nature 397, $50-53$

Muellerova, D., Krajcikova, D., Barak, I., 2009. Interactions between Bacillus subtilis early spore coat morphogenetic proteins. Fems Microbiol. Lett. 299, 74-85.

Nicholson, W.L., Munakata, N., Horneck, G., Melosh, H.J., Setlow, P., 2000. Resistance of Bacillus endospores to extreme terrestrial and extraterrestrial 
environments. Microbiol. Mol. Biol. Rev. 64, 548-572.

Ozin, A.J., Henriques, A.O., Yi, H., Moran, C.P., 2000. Morphogenetic proteins SpoVID and SafA form a complex during assembly of the Bacillus subtilis spore coat. J. Bacteriol. 182, 1828-1833.

Ozin, A.J., Samford, C.S., Henriques, A.O., Moran, C.P., 2001. SpoVID guides SafA to the spore coat in Bacillus subtilis. J. Bacteriol. 183, 3041-3049.

Piggot, P.J., Coote, J.G., 1976. Genetic aspects of bacterial endospore formation. Bacteriol. Rev. 40, 908-962.

Plomp, M., Carroll, A.M., Setlow, P., Malkin, A.J., 2014. Architecture and assembly of the Bacillus subtilis spore coat. Plos One 9, e108560.

Plomp, M., Rice, M.K., Wagner, E.K., McPherson, A., Malkin, A.J., 2002. Rapid visualization at high resolution of pathogens by atomic force microscopy: structural studies of herpes simplex virus-1. Am. J. Pathol. 160, 1959-1966.

Pogliano, K., Harry, E., Losick, R., 1995. Visualization of the subcellular location of sporulation proteins in Bacillus subtilis using immunofluorescence microscopy. Mol. Microbiol. 18, 459-470.

Qiao, H., Krajcikova, D., Liu, C., Li, Y., Wang, H., Barak, I., Tang, J., 2012. The interactions of spore-coat morphogenetic proteins studied by single-molecule recognition force spectroscopy. Chem. Asian J. 7, 725-731.

Qiao, H., Krajcikova, D., Xing, C., Lu, B., Hao, J., Ke, X., Wang, H., Barak, I., Tang, J., 2013. Study of the interactions between the key spore coat morphogenetic proteins CotE and SpoVID. J. Struct. Biol. 181, 128-135. 
Ramamurthi, K.S., Clapham, K.R., Losick, R., 2006. Peptide anchoring spore coat assembly to the outer forespore membrane in Bacillus subtilis. Mol. Microbiol. 62, $1547-1557$.

Riener, C.K., Stroh, C.M., Ebner, A., Klampfl, C., Gall, A.A., Romanin, C., Lyubchenko, Y.L., Hinterdorfer, P., Gruber, H.J., 2003. Simple test system for single molecule recognition force microscopy. Anal. Chim. Acta 479, 59-75.

Shao, Z.F., Mou, J., Czajkowsky, D.M., Yang, J., Yuan, J.Y., 1996. Biological atomic force microscopy: what is achieved and what is needed. Adv. Phys. 45, 1-86.

Tang, J., Ebner, A., Kraxberger, B., Leitner, M., Hykollari, A., Kepplinger, C., Grunwald, C., Gruber, H.J., Tampe, R., Sleytr, U.B., Ilk, N., Hinterdorfer, P., 2009. Detection of metal binding sites on functional S-layer nanoarrays using single molecule force spectroscopy. J. Struct. Biol. 168, 217-222.

Wang, K.H., Isidro, A.L., Domingues, L., Eskandarian, H.A., McKenney, P.T., Drew, K., Grabowski, P., Chua, M.-H., Barry, S.N., Guan, M., Bonneau, R., Henriques, A.O., Eichenberger, P., 2009. The coat morphogenetic protein SpoVID is necessary for spore encasement in Bacillus subtilis. Mol. Microbiol. 74, 634-649.

Wildling, L., Rankl, C., Haselgruebler, T., Gruber, H.J., Holy, M., Newman, A.H., Zou, M.-F., Zhu, R., Freissmuth, M., Sitte, H.H., Hinterdorfer, P., 2012. Probing binding pocket of serotonin transporter by single molecular force spectroscopy on living cells. J. Biol. Chem. 287, 105-113.

Wollschlaeger, K., Gaus, K., Koernig, A., Eckel, R., Wilking, S.-D., McIntosh, M., Majer, Z., Becker, A., Ros, R., Anselmetti, D., Sewald, N., 2009. Single-molecule 
experiments to elucidate the minimal requirement for DNA recognition by transcription factor epitopes. Small 5, 484-495.

Zhang, J., Fitzjames, P.C., Aronson, A.I., 1993. Cloning and charcterization of a cluster of genes encoding polypeptides present in the insoluble fraction of the spore coat of Bacillus subtilis. J. Bacteriol. 175, 3757-3766.

Zhang, J., Chtcheglova, L.A., Zhu, R., Hinterdorfer, P., Zhang, B., Tang, J., 2014. Nanoscale organization of human GnRH-R on human bladder cancer cells. Anal. Chem. 86, 2458-2464.

Zheng, L.B., Donovan, W.P., Fitzjames, P.C., Losick, R., 1988. Gene encoding a morphogenic protein required in the assembly of the outer coat of the Bacillus subtilis endospore. Genes Dev. 2, 1047-1054.

Zhu, R., Howorka, S., Proell, J., Kienberger, F., Preiner, J., Hesse, J., Ebner, A., Pastushenko, V.P., Gruber, H.J., Hinterdorfer, P., 2010. Nanomechanical recognition measurements of individual DNA molecules reveal epigenetic methylation patterns. Nat. Nanotechnol. 5, 788-791.

Zilhao, R., Serrano, M., Isticato, R., Ricca, E., Moran, C.P., Henriques, A.O., 2004. Interactions among $\mathrm{CotB}, \mathrm{Cot} \mathrm{G}$, and $\mathrm{Cot} \mathrm{H}$ during assembly of the Bacillus subtilis spore coat. J. Bacteriol. 186, 1110-1119. 


\section{Figure Legends}

Fig. 1. (a) Schematic representation of AFM force measurements with a CotZ-attached tip and the CotE-attached silicon substrate. (b) Typical force-distance cycle for a recognition event between CotE and CotZ. Recognition signal disappears in the control experiment, which uses an AFM tip functionalized only with the PEG linker (inset). (c) Distribution of the unbinding forces for the interaction of CotE and CotZ (black, solid line) and the PEG linker functionalized AFM tip and CotE in the control experiment (red, dashed line).

Fig. 2. The unbinding forces for the interaction between $\operatorname{CotE}$ and $\operatorname{Cot} Z$ were plotted against the logarithm of the loading rate.

Fig. 3. AFM height images of $\operatorname{CotE}\left(a_{1}\right)$ and $\operatorname{Cot} Z\left(b_{1}\right)$ individually assembling on the silicon substrates. The height scales are from -7.5 to $7.5 \mathrm{~nm}$ and -5.0 to $5.0 \mathrm{~nm}$ for panels $\left(a_{1}\right)$ and $\left(b_{1}\right)$, respectively. $\left(a_{2}\right)$ Higher-magnification view of CotE from a portion of panel $\left(a_{1}\right)$, with a height scale of -7.6 to $7.6 \mathrm{~nm}$. $\left(b_{2}\right)$ Higher-magnification view of CotZ from a portion of panel $\left(b_{1}\right)$, with a height scale of -4.3 to $4.3 \mathrm{~nm}$. Cross-sections obtained along the white lines. White and black arrows indicated meshes and filaments formed by the proteins, respectively.

Fig. 4. Typical AFM images of the complex of CotE with CotZ formed at different molar ratios: $\left(a_{1}\right) \operatorname{CotE}: \operatorname{CotZ} 3: 1$, with a height scale of -4.0 to $4.0 \mathrm{~nm},\left(\mathrm{~b}_{1}\right) \operatorname{CotE}: \operatorname{Cot} Z$ 
2:1, with a height scale of -2.0 to $2.0 \mathrm{~nm},\left(\mathrm{c}_{1}\right) \operatorname{CotE}: \operatorname{CotZ} 1: 2$, with a height scale of -4.0 to $4.0 \mathrm{~nm}$ and $\left(\mathrm{d}_{1}\right) \operatorname{CotE}: \operatorname{CotZ} 1: 3$, with a height scale of -7.5 to $7.5 \mathrm{~nm}$. Right panels $\left(a_{2}, b_{2}, c_{2}\right.$ and $\left.d_{2}\right)$ are the corresponding magnification views of a portion of left panels, with the height scales of -5.1 to $5.1 \mathrm{~nm},-2.0$ to $2.0 \mathrm{~nm},-3.5$ to $3.5 \mathrm{~nm}$ and -7.0 to $7.0 \mathrm{~nm}$, respectively. Cross-sections obtained along the white lines. White and black arrows indicated meshes and filaments formed by the proteins, respectively. 
(a)

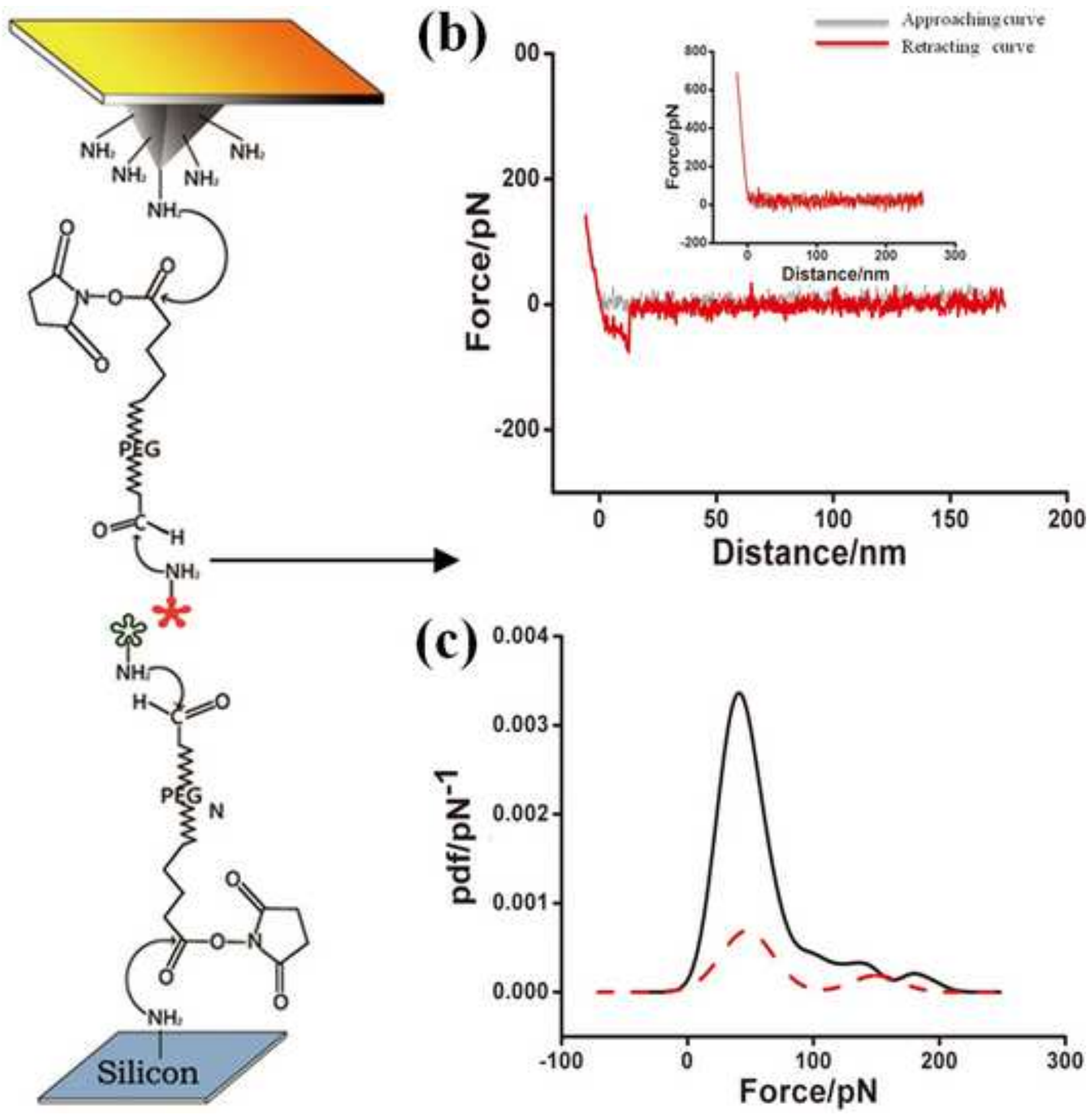




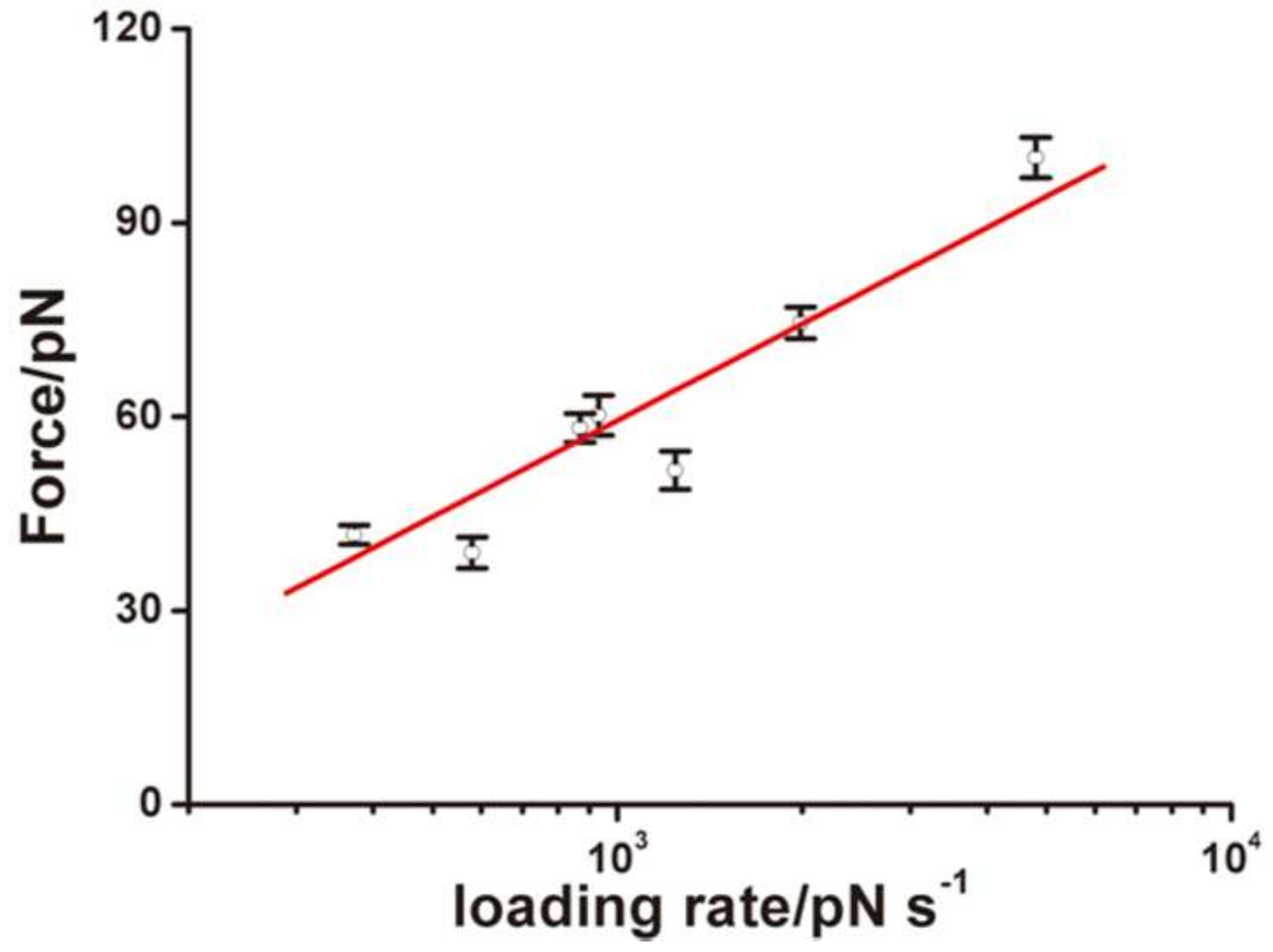



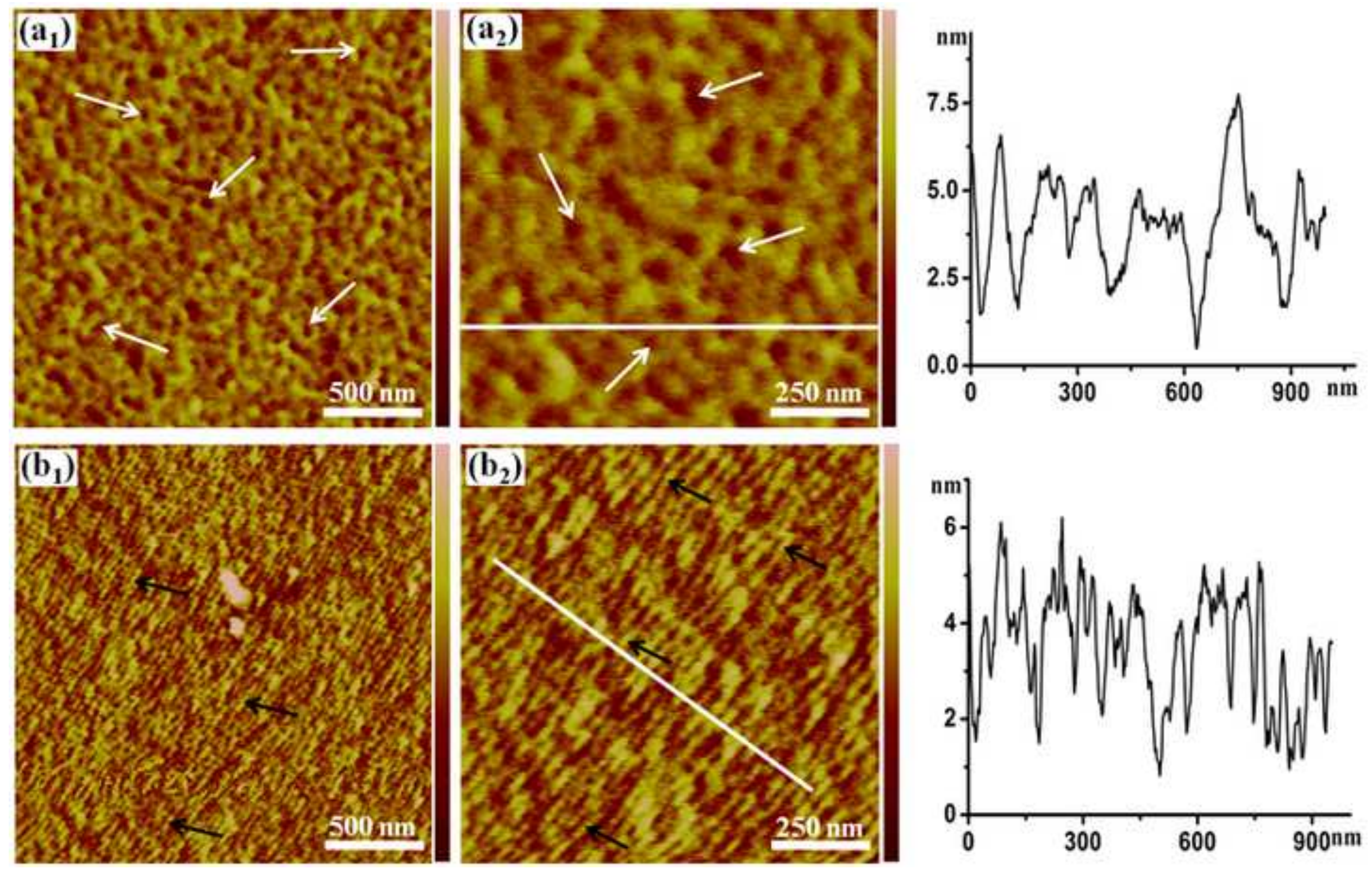

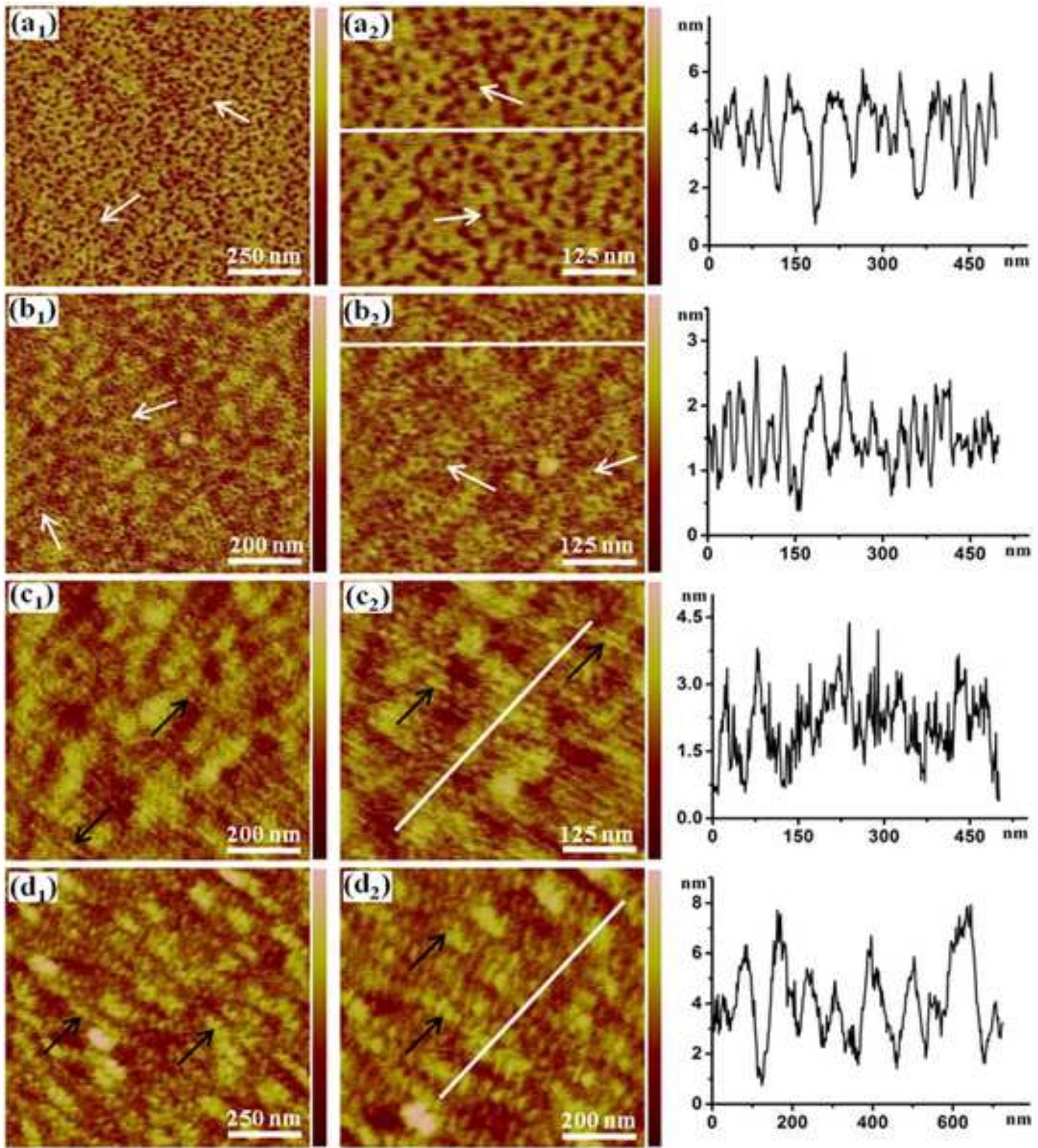